\title{
VIDEO IMAGES AND UNDULATORY MOVEMENT EQUATION OF PANGASIUS SANITWONGSEI'S CAUDAL FIN OF STEADY SWIMMING FISH
}

\author{
A. S. VAGHEFI ${ }^{1} \&$ M. ABBASPOUR ${ }^{2}$ \\ ${ }^{1}$ Department of Mechanical Engineering, Shahr-e-Rey Branch, Islamic Azad University (IAU), Iran. \\ ${ }^{2}$ Department of Mechanical Engineering, Sharif University of Technology, Iran.
}

\begin{abstract}
Experimental hydrodynamics imaging of four Pangasius sanitwongsei were considered. A quantitative characterization of caudal fin is presented in this article. Steady swimming of four $P$. sanitwongsei with different total length was studied experimentally and taped by high-speed digital video, and undulatory movement of each fish at different velocity was revealed. The pattern of body undulatory movement of the fish was drawn from the video images. Three main factors that determine the fish swimming behavior are Reynolds number, Strouhal number and shape. In this study, Lf/L was chosen as a characteristic of shape, where Lf was the distance from the start of the head to the end of the head. This is a major point and displays less variation of head to the more variation of the body and caudal fin. $\mathrm{L}$ is the length of the fish body. The relationship between Reynolds number and Strouhal number of four $P$. sanitwongsei with different Lf/L were studied here. Then, the relationship between effective non-dimensional parameters in thrust force and kinematic parameters was found. As a result, an experimental equation was formulated. This equation indicates that, as much as the ratio of the end part of fish with high undulatory movement (body and caudal fin) to the total length goes up, the ratio of amplitude to the total length increases. Consequently, there was an increase in displacement and thrust force also. Then, undulatory movement equation of fish swimming was calculated by fitting a second-order function that describes wave amplitude of this type of fish. All the finding in these researches could be applied to design
\end{abstract} a robotic fish.

Keywords: Movement equation, fish swimming, robot fish, video image, Pangasius, undulatory.

\section{INTRODUCTION}

In nature, fish are propelled by the undulatory motion of their body and have gained marvelous swimming ability over many years of evolution. For more than decades, scientists have marveled at the exquisite way in which fish swim and accelerate [1]. Tuna move with high speed, pike accelerate at the moment, and eels can swim into every hole [2]. From a mechanics appearance, their swimming raises questions: how wonderful they are as swimmers? Are they more practical than a submarine vehicle? [1]. Many researchers have been trying to find the answer to these questions. Fish swimming has been studied widely using experimental, numerical and theoretical techniques. Experimental studies have been applied on the robot fish, fish and biomimetic foils. In 1994, the first robot fish RoboTuna was built by the scientists at the MIT [3]. In 1998, Anderson et al. researched the flow around two-dimensional flapping foils as function of the Strouhal number and the angle of attack. The Strouhal number is defined as $\mathrm{St}=\mathrm{f} \cdot \mathrm{A} / \mathrm{u}$, where A denotes the characteristic width, $\mathrm{f}$ the frequency of movement, and $u$ the velocity of the foil. They presented wake patterns as a function of the angle of attack and the Strouhal number [4]. In 2002, Lauder et al. studied hydrodynamics of fish swimming with digital particle image velocimetry that allowed experimental analysis of force magnitude and direction. They examined fin function in four ray-finned fish clades: sunfish, sturgeon, mackerel and trout [5]. In 2004, Gilbert characterized three-dimensional kinematics of pectoral fin swimming in bluegill sunfish quantitatively. Fish swimming at several speeds was taped in a flow tank that is $45 \mathrm{~cm}$ long by $18 \mathrm{~cm}$ width and $18 \mathrm{~cm}$ height. Flow patterns were visualized with the high-resolution (1024 × 1024 pixels) and high-speed (500 fps) camera [6]. In 2006, Lauder and 
Madden revealed 16 results of new experimental research on the fluid dynamics, mechanics, control and kinematics of fish movement that summarize the recent works on fish biomechanics [7]. In 2006, Bejan and Marden showed swimming, running and flying can be described by a single theory that they use optimal energy. They compared theoretical predictions of different animals such as mammals, birds and fish. They achieved the relationship between velocity, frequency, force and body mass of the animals [8]. When the fish move forward, water will go out of the path and the only way the water must go is up. In this process, the needed work to move the water vertically is the weight of fish multiplying body length of fish. Also there is a drag force that prevents the forward movement of fish. So fish do work horizontally, that is, drag force multiplying body length of fish [9]. In 2007, Standen and Lauder showed that the dorsal fins generate vortices that are encountered by the caudal fin. These vortices can go up the thrust generated by caudal fins [10]. In 2008, Masalo et al. studied fish swimming using acoustical Doppler velocimetry (ADV) techniques. Turbulence was evaluated using the RMS velocity as an indicator of fish movement. They revealed that the relationship between RMS and density was linear [11]. In 2011, Lauder indicated 10 questions and technical approaches about swimming hydrodynamics that can serve as a starting point for thinking about new perspective for the next research [12].

In this study, the flow patterns of fish swimming were visualized and the kinematic parameters of four Pangasius sanitwongse $i$ were determined, which in turn it redound to define the equation of fish movement.

\section{FISH SWIMMING MODE}

There are several features in fish swimming that seem to be necessary to consider for designing robotic devices for propulsion purposes in submarine. Among them is swimming mode that is of a crucial importance for every scientists [13]. Taking into account fish motion, one can classifies fish into groups based on fin functions. Accordingly, fish fins can be divided into various categories based on the place where fin is located such as caudal fin, which is at the end of fish body; dorsal fin, which is on the top of fish body; two pectoral fins, which are on each side of their body; and pelvic and anal fins, which are beneath fish body. Each of these fins has a specific mechanism and motion (Fig. 1). Those fish swim with their median fins (dorsal and anal) and paired fins (pectoral and pelvic) are termed MPF (median and paired fin) swimmers and those that use primarily their body and caudal fin are called BCF (body and caudal fin) swimmers [14].

Increasing maneuverability and propulsive efficiency, MPF is used at slow speeds, while BCF movement can increase thrust force and accelerations [10]. Lindsey classifies different modes of propulsive movements of fish into four groups [15]: anguilliform, subcarangiform, carangiform and thunniform. In anguilliform mode, the whole body functions as thrust force. In the second mode, subcarangiform, the rear half of the body contributes to propulsive mechanism. In carangiform mode, the last third of the body has an undulatory movement. Finally in thunniform mode, the tail movement produces the thrust force. There are also other classifications to study fish movement. By

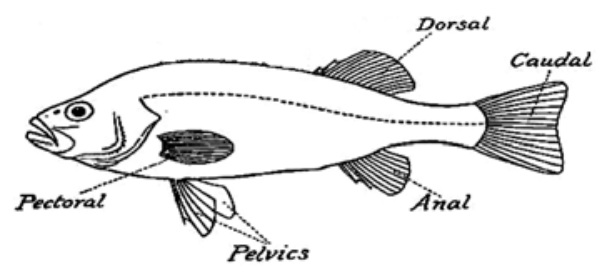

Figure 1: Median and paired fins. 
comparing caudal fin structure, fish are classified into two groups: fish with a forked tail, which create a series of vortex ring, and fish without forked tail. The caudal fins generate thrust forces that are different in mentioned groups and the mechanical performance is also different [16].

However, this study aims at examining BCF mechanism of four $P$. sanitwongsei.

\section{MATERIALS AND METHODS}

There are some major non-dimensional parameters in fish movement. In 1988, Webb identified three main factors in fish swimming: Reynolds number, Strouhal number and shape [17].

Reynolds number is defined as the ratio of inertial forces to viscous forces. This number is formulated as $\mathrm{Re}=\mathrm{u} \cdot \mathrm{L} / \mathrm{v}$, in which $\mathrm{u}$ is the velocity of fish movement, $\mathrm{L}$ is the length of the fish and $v$ is kinematic viscosity of water. Strouhal number is formulated as $\mathrm{St}=\mathrm{f} \cdot \mathrm{A} / \mathrm{u}$ is the other non-dimensional parameter in fish movement. In this equation, $\mathrm{f}$ is the tail-beat frequency, $\mathrm{A}$ is the amplitude of undulatory movement of tail and $u$ is the average velocity of fish $[1,13,17]$. The Strouhal number is defined as the ratio of unsteady to inertial force [19]. There is also another commonly used nondimensional parameter, that is $\mathrm{Lf} / \mathrm{L}$. In this study, $\mathrm{Lf} / \mathrm{L}$ is applied as a characteristic of shape, where Lf is the distance from start of the head to the end of the head. This is a key parameter that shows the less variation of head to the more variation of the body and caudal fin. Furthermore, $\mathrm{L}$ is the length of fish body, L-Lf is the major deformable length of the body (working as thrust generating) in the rear, and the position $\mathrm{x}=\mathrm{Lf}$ provides the minimum amplitude along the entire body (Fig. 2) [18].

Moreover, in this experiment, $P$. sanitwongsei (Fig. 3) with several total lengths is studied in a glassy tank with $90 \mathrm{~cm}$ length, $30 \mathrm{~cm}$ width, and $45 \mathrm{~cm}$ height. In the experiment, the water was kept at the temperature $20^{\circ} \mathrm{C}$ and the kinematic viscosity was $v=1.005 \times 10^{-6}\left(\mathrm{~m}^{2} / \mathrm{s}\right)$. As mentioned before, one of the important factors for fish movement is its shape. Here, $\mathrm{Lf} / \mathrm{L}$ was chosen for mainly

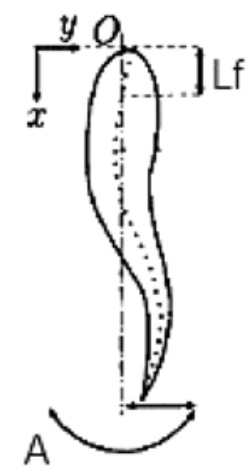

Figure 2: Lf in typical fish movement [18].

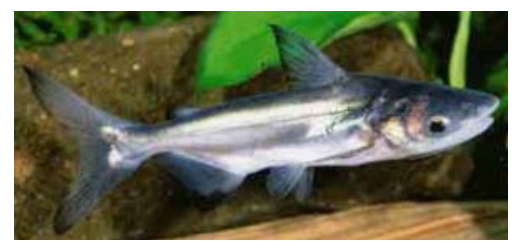

Figure 3: Pangasius sanitwongsei that was studied in this work. 


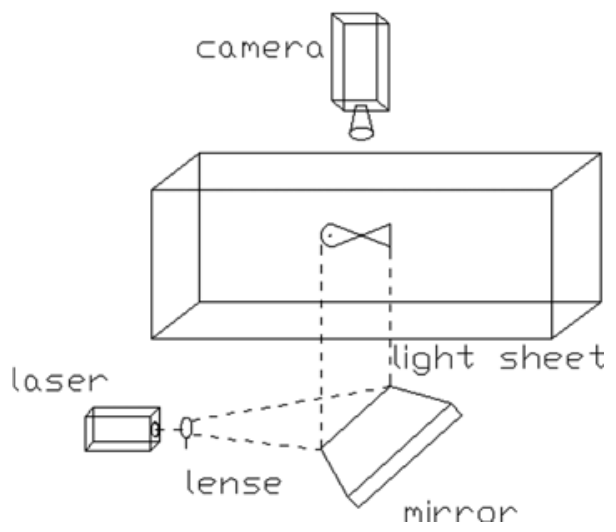

Figure 4: Schematic of the experimental set up. Images of fish swimming are obtained with the high speed video camera. A planar slice of the flow is illuminated with a $100 \mathrm{~mW}$ Nd Yag laser $(\lambda=532 \mathrm{~nm})$ aimed at the $45^{\circ}$ front-surface mirror positioned below the flow tank.

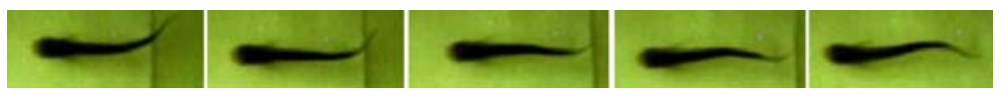

Figure 5: Undulatory movement of caudal fin is presented. Five original images of Pangasius sanitwongsei at $\Delta \mathrm{t}=0.02 \mathrm{~s}$ by $\mathrm{L}=9 \mathrm{~cm}$. Half of wavelength is represented at $80 \mathrm{msec}$.

determining fish shape. To do so, a range of fishes with different body length are examined: $12 \mathrm{~cm}$, $9 \mathrm{~cm}, 7.5 \mathrm{~cm}$ and $5.8 \mathrm{~cm}$, with $\mathrm{Lf} / \mathrm{L} 0.250,0.280,0.270$ and 0.260 , respectively.

To estimate the fish movement behavior, images are videotaped by a Cube 3 camera at $2500 \mathrm{fps}$ at maximum resolution, with maximum frame capture rate of 120,000 fps.

The experimental set-up is shown in Fig. 4. A planar slice of the flow is illuminated with a $100 \mathrm{~mW}$ laser. Flow pattern is revealed through polystyrene small particles $(40-60 \mu \mathrm{m})$. Fish movement is visualized a number of times for each fish and ultimately some applicable frames is chosen. Figure 5 demonstrates five frames of $P$. sanitwongsei with $9 \mathrm{~cm}$ body length and $\Delta \mathrm{t}=20 \mathrm{msec}$.

\section{RESULTS}

In this study, undulatory movements of four P. sanitwongsei were examined and recorded. Among all images, two more proper pictures were selected for each case. Figure 5 demonstrates five sequential images of $P$. sanitwongse $i$ at $\Delta \mathrm{t}=20 \mathrm{msec}$ by total length $9 \mathrm{~cm}$. These images present undulatory movement of fish tail while it is passing half of wave length. Amplitude, frequency and velocity of fish movement were found with these video images. Then, two dimensionless parameters Strouhal number and Reynolds number were obtained. Table 1 shows the summary of fish movement variables of four $P$. sanitwongsei with different total length. These parameters were found from experimental results. The pattern of undulatory movement of four $P$. sanitwongse $i$ was extracted from above images. These patterns are presented in Figs 6-9.

All measurements tabulated from experimental results of Pangasius sanitwongsei. f indicates frequency, A indicates amplitude and $\mathrm{u}$ indicates velocity. Velocity and amplitude have been revealed as a proportion of total length. 
Table 1: Summary of fish movement variables of Pangasius sanitwongsei.

\begin{tabular}{|c|c|c|c|c|c|c|c|c|}
\hline & $\mathrm{L}(\mathrm{cm})$ & $\operatorname{Lf}(\mathrm{cm})$ & $\mathrm{A}(\mathrm{cm})$ & $\mathrm{f}(1 / \mathrm{s})$ & $\mathrm{u}(\mathrm{cm} / \mathrm{s})$ & $\mathrm{Lf} / \mathrm{L}$ & $\mathrm{St}$ & $\operatorname{Re}$ \\
\hline \multirow[t]{3}{*}{ No.1 } & 12 & 3.00 & $0.28 \mathrm{~L}$ & 1.67 & $0.98 \mathrm{LT}^{-1}$ & 0.250 & 0.47 & 14042 \\
\hline & & & $0.19 \mathrm{~L}$ & 1.43 & $0.81 \mathrm{LT}^{-1}$ & & 0.33 & 11678 \\
\hline & & & $0.22 \mathrm{~L}$ & 1.67 & $0.89 \mathrm{LT}^{-1}$ & & 0.41 & 12800 \\
\hline \multirow[t]{3}{*}{ No.2 } & 9 & 2.52 & $0.11 \mathrm{~L}$ & 8.33 & $5.38 \mathrm{LT}^{-1}$ & 0.280 & 0.17 & 43397 \\
\hline & & & $0.33 \mathrm{~L}$ & 6.25 & $5.67 \mathrm{LT}^{-1}$ & & 0.36 & 45672 \\
\hline & & & $0.13 \mathrm{~L}$ & 8.33 & $5.37 \mathrm{LT}^{-1}$ & & 0.21 & 43379 \\
\hline \multirow[t]{3}{*}{ No.3 } & 7.5 & 2.03 & $0.12 \mathrm{~L}$ & 5.00 & $2.60 \mathrm{LT}^{-1}$ & 0.270 & 0.25 & 14366 \\
\hline & & & $0.15 \mathrm{~L}$ & 8.33 & $3.46 \mathrm{LT}^{-1}$ & & 0.35 & 19343 \\
\hline & & & $0.13 \mathrm{~L}$ & 5.00 & $3.22 \mathrm{LT}^{-1}$ & & 0.21 & 18037 \\
\hline \multirow[t]{3}{*}{ No.4 } & 5.8 & 1.51 & $0.23 \mathrm{~L}$ & 3.12 & $1.96 \mathrm{LT}^{-1}$ & 0.260 & 0.37 & 6568 \\
\hline & & & $0.31 \mathrm{~L}$ & 4.17 & $2.85 \mathrm{LT}^{-1}$ & & 0.46 & 9569 \\
\hline & & & $0.20 \mathrm{~L}$ & 3.55 & $2.15 \mathrm{LT}^{-1}$ & & 0.33 & 7260 \\
\hline
\end{tabular}

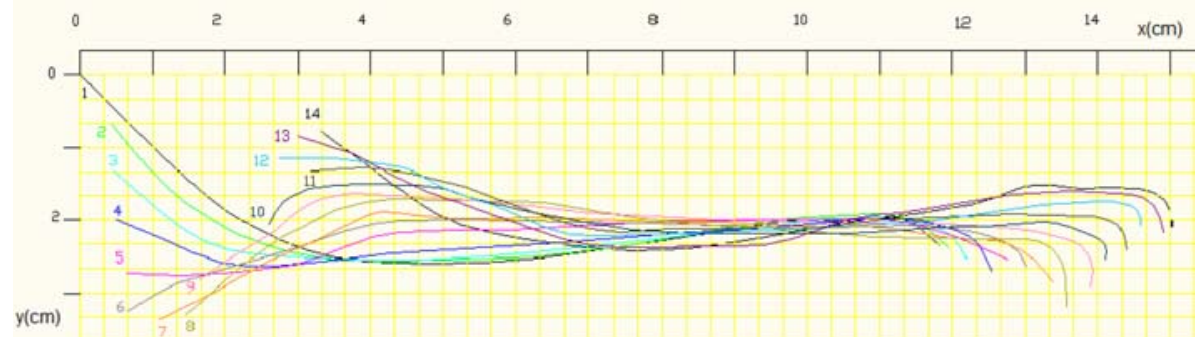

Figure 6: Patterns of body undulatory movement of Pangasius sanitwongsei at $\Delta \mathrm{t}=50 \mathrm{msec}$ by $\mathrm{L}=12 \mathrm{~cm}$.

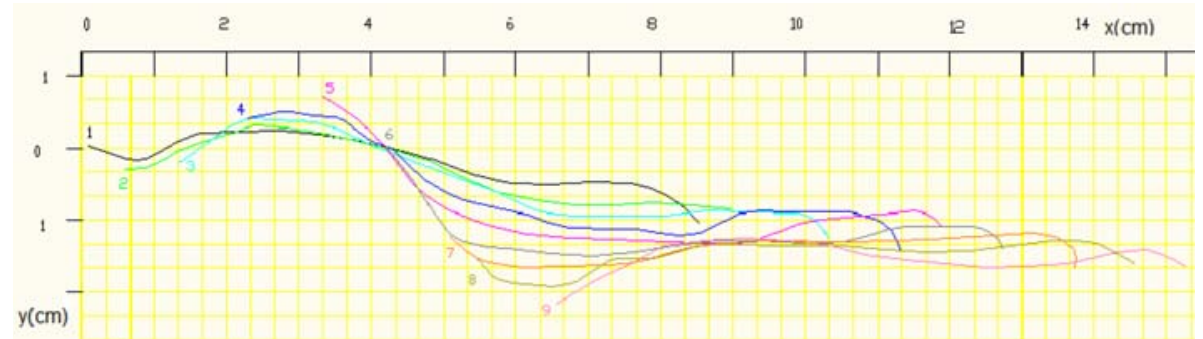

Figure 7: Patterns of body undulatory movement of Pangasius sanitwongsei by $\mathrm{L}=9 \mathrm{~cm}$ at $\Delta \mathrm{t}=20 \mathrm{msec}$.

Bejan and Marden presented the optimal swimming speed as $V_{\text {opt }} \sim g^{1 / 2} \rho^{-1 / 6} \mathrm{M}^{1 / 6}$, where $g$ is the gravitational acceleration, $\rho$ is the body density of fish and $M$ is the body mass of fish [8]. They revealed swimming speed varied from 0.1 to $0.7 \mathrm{~m} / \mathrm{s}$ approximately for the fish that have body mass between 0.1 and 1 kilogram. In this study, the swimming speed varied from 0.096 to $0.51 \mathrm{~m} / \mathrm{s}$. So 


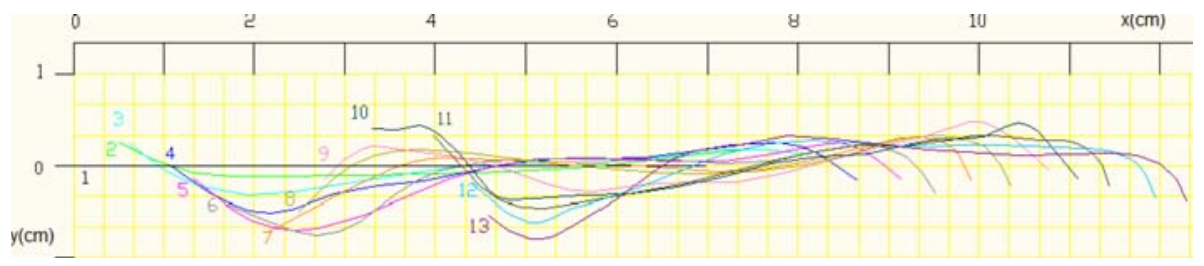

Figure 8: Patterns of body undulatory movement of Pangasius sanitwongsei by $\mathrm{L}=7.5 \mathrm{~cm}$ at $\Delta \mathrm{t}=20 \mathrm{msec}$.

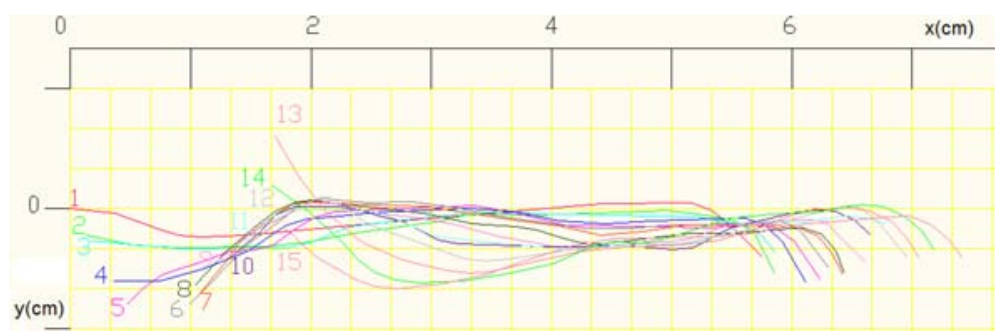

Figure 9: Patterns of body undulatory movement of Pangasius sanitwongsei by $\mathrm{L}=5.8 \mathrm{~cm}$ at $\Delta \mathrm{t}=20 \mathrm{msec}$.

there is an acceptable consistency between these results (Table 1) and the theoretical predictions of Bejan and Marden.

Bejan and Marden presented the optimal undulating frequency of fish as $\mathrm{f}_{\text {opt }} \sim \mathrm{V}_{\text {opp }} / \mathrm{L} \sim \mathrm{g}^{1 / 2} \rho^{1 / 6}$ $\mathrm{M}^{-1 / 6}$, where $\mathrm{L}$ is the distance traveled, $\mathrm{g}$ is the gravitational acceleration, $\rho$ is the body density of fish and $\mathrm{M}$ is the body mass of fish [8].

They showed frequency changed from 2 to $9 \mathrm{~Hz}$ approximately for the fish that have body mass between 0.1 and 1 kilogram. Table 1 presents frequency of swimming fish varied from 1.43 to 8.33 in this work, which was supported by the result of Bejan and Marden.

Figure 6 reveals body outlines extracted from fish movement of $P$. sanitwongse $i$ with total length, $\mathrm{L}=12 \mathrm{~cm}$. This image presents one tail-beat cycle recorded at intervals of $50 \mathrm{~ms}$. As shown in this figure, fish movement variables such as frequency, amplitude and velocity could be determined. In addition, this figure represents that the amplitude of this wave increases dramatically near the tail while it is very low near the head.

Figure 7 shows the body outlines taken from movies of fish swimming with $\mathrm{L}=9 \mathrm{~cm}$. This image presents one tail-beat cycle that was recorded at intervals of $20 \mathrm{~ms}$. Body outlines of $P$. sanitwongsei with total length $\mathrm{L}=7.5 \mathrm{~cm}$ and $5.8 \mathrm{~cm}$ are shown in Figs 8 and 9, respectively. Frequency, velocity and amplitude are found from these images.

Figure 10 presents the undulatory movement of caudal fin of four $P$. sanitwongse $i$ in steady swimming. The time interval between sequential plotted tail situations was $20 \mathrm{msec}$. The total experimental recording time was $200 \mathrm{msec}$. This picture reveals that undulatory movement of caudal fin of $P$. sanitwongse $i$ is similar to a sinusoidal form. Moreover, it means that undulatory movement of caudal fin becomes closer to a complete sinusoidal form, as $\mathrm{Lf} / \mathrm{L}$ raises. In order to clear, undulatory movements of caudal fin of four P. sanitwongse $i$ with different $\mathrm{Lf} / \mathrm{L}, 0.250,0.260,0.270$ and 


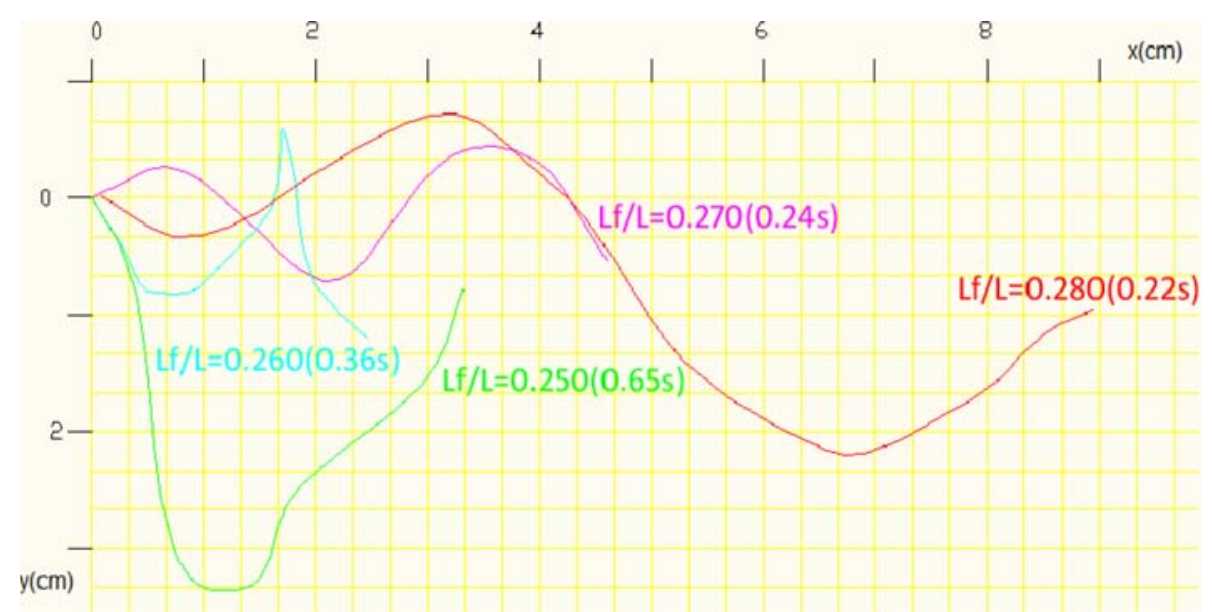

Figure 10: Undulatory movement of caudal fin of four Pangasius sanitwongsei with different Lf/L. The number in parenthesis shows total experimental recording time for each fish.

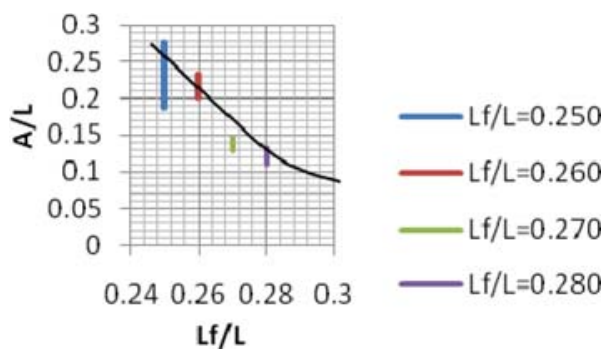

Figure 11: The relationship between $\mathrm{A} / \mathrm{L}$ and $\mathrm{Lf} / \mathrm{L}$.

0.280 , were considered. As presented here, even though the capture time for lower $\mathrm{Lf} / \mathrm{L}$ is almost three times of the case of higher $\mathrm{Lf} / \mathrm{L}$, its behavior is not in a sinusoidal form.

\section{DISCUSSION}

The body behavior of four P. sanitwongsei was studied in this experimental test. Fish movement was taped with a high-speed digital video system and the undulatory movement of caudal fin was drawn. Figures 6 to 9 reveal body outlines taken from fish swimming of $P$. sanitwongsei by different total length. While the amplitude of this wave increases significantly near the tail, it is very low near the head. As it has been presented, Re has found in the region of adult fish movement $\left(10^{3}<\operatorname{Re}<5 \times 10^{6}\right)$ where inertial forces are great and viscous forces are neglected. In subcarangiform and carangiform modes, for high speed movement $\left(10^{4}<\operatorname{Re}<10^{6}\right)$ thrust is optimal for a specific realm of St, where $0.25<\mathrm{St}<0.40$ [13].

The relationship between $\mathrm{A} / \mathrm{L}$ and $\mathrm{Lf} / \mathrm{L}$ of four fish is shown in Fig. 11. A/L is the ratio of amplitude to total length. This figure reveals a decrease in $\mathrm{A} / \mathrm{L}$ while $\mathrm{Lf} / \mathrm{L}$ increases. In fact, if the ratio of the end part of the fish with high undulatory movement (body and caudal fin) to the total length goes up, the ratio of amplitude to the total length would increase; therefore, thrust force would also increase. 
An experimental equation was formulated, which represents the relationship between A/L and $\mathrm{Lf} / \mathrm{L}$ of $P$. sanitwongse $i$ in steady swimming, based on this figure [19]:

$$
(\mathrm{A} / \mathrm{L})=\mathrm{p}(\mathrm{Lf} / \mathrm{L})^{\mathrm{k}},
$$

where $\mathrm{k}=-7.109$ and $\mathrm{p}=1.464$. $\mathrm{k}$ and $\mathrm{p}$ are named as I.A. coefficient.

Figure 12 reveals the relationship between the $\mathrm{A} / \mathrm{L}$ and $\mathrm{u} / \mathrm{L}$ of four $P$. sanitwongse $i$ with different $\mathrm{Lf} / \mathrm{L} . \mathrm{u} / \mathrm{L}$ is the ratio of fish swimming velocity to total length. In these studies, fish swimming velocity varied from $0.8 \mathrm{LS}^{-1}$ to $5.7 \mathrm{LS}^{-1}$. This figure shows at the constant $\mathrm{A} / \mathrm{L}, \mathrm{u} / \mathrm{L}$ increased while $\mathrm{Lf} / \mathrm{L}$ was increasing.

Figure 13 represents the relationship between Reynolds number and Strouhal number of four P. sanitwongsei with different Lf/L. For these studied cases, Re varied from $6.5 \times 10^{3}$ to $4.5 \times 10^{4}$ and St varied from 0.17 to 0.47 .

Figure 14 reveals the relationship between fish swimming velocity (u) and frequency of undulatory movement (f) of four P. sanitwongsei with different Lf/L. Figure 15 shows the relationship between swimming velocity (u) and frequency (f) for different materials at. Ziegler's work in 2006 [20]. Red curve ( $\square$ ) shows flexible material, blue curve $(\Delta)$ shows soft material and the yellow one (o) shows hard material. Comparing Figure 14 and 15, it seems a clear peak for each fish must indicate the optimal frequency in Fig. 14. To achieve more accurate result, it is suggested to study fish swimming in higher latitude. However, Fig. 14 reveals that fish swimming velocity (u) and frequency of undulatory movement (f) increased while $\mathrm{Lf} / \mathrm{L}$ was increasing.

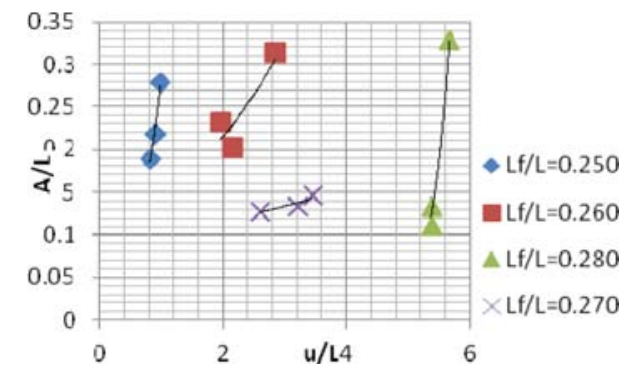

Figure 12: The relationship between $\mathrm{A} / \mathrm{L}$ and $\mathrm{u} / \mathrm{L}$ for different $\mathrm{Lf} / \mathrm{L}$.

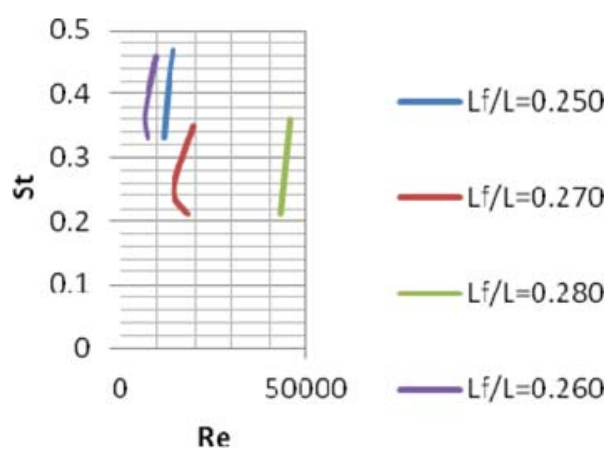

Figure 13: The relationship between St and Re for different Lf/L. 


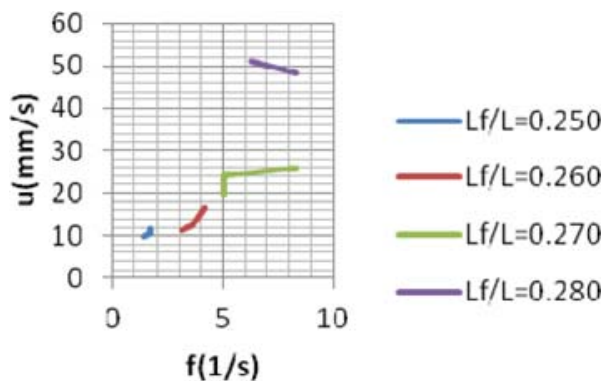

Figure 14: The relationship between fish swimming velocity (u) and frequency (f) for different Lf/L.

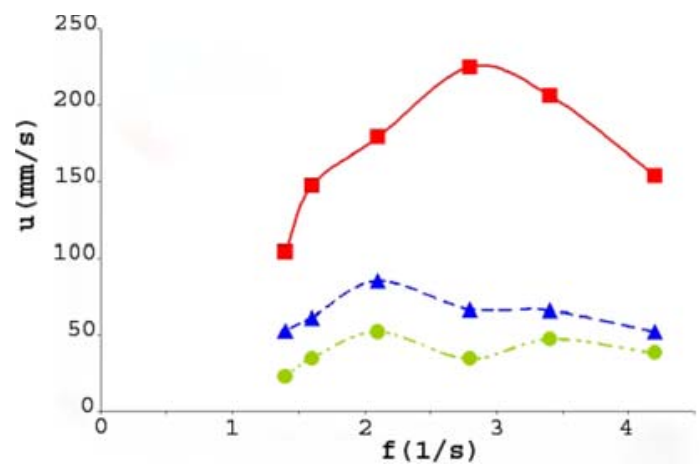

Figure 15: The relationship between swimming velocity (u) and frequency (f) for different materials. Red curve shows flexible material, blue curve shows soft material and the yellow one shows hard material [20].

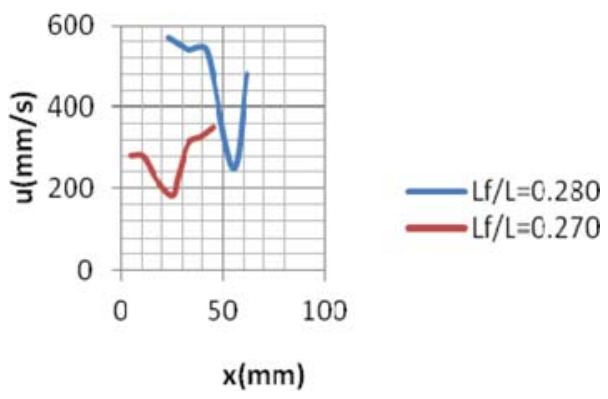

Figure 16: The relationship between velocity and $\mathrm{x}$ axis for different $\mathrm{Lf} / \mathrm{L}$.

Figure 16 represents the relationship between fish swimming velocity and $\mathrm{x}$ axis for two $P$. sanitwongsei with different $\mathrm{Lf} / \mathrm{L}$. It shows a sinusoidal form for variation $\mathrm{u}$ and $\mathrm{x}$. Figure 17 represents the relationship between fish swimming velocity and $\mathrm{x}$ axis for the others $P$. sanitwongsei with $\mathrm{Lf} / \mathrm{L}=0.250$ and 0.260 . The variation $u$ and $x$ is partly similar to Fig. 16 . 
104 A. S. Vaghefi \& M. Abbaspour, Int. J. of Design \& Nature and Ecodynamics. Vol. 9, No. 2 (2014)

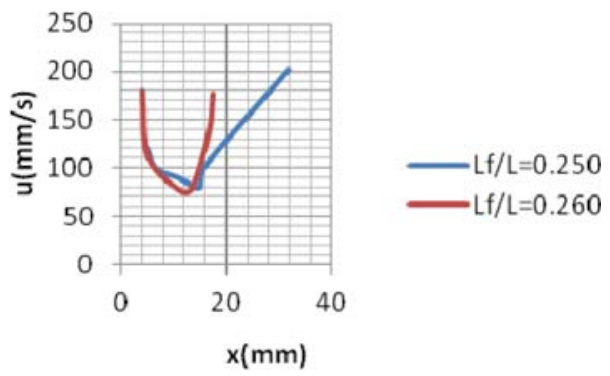

Figure 17: The relationship between velocity and $\mathrm{x}$ axis for different $\mathrm{Lf} / \mathrm{L}$.

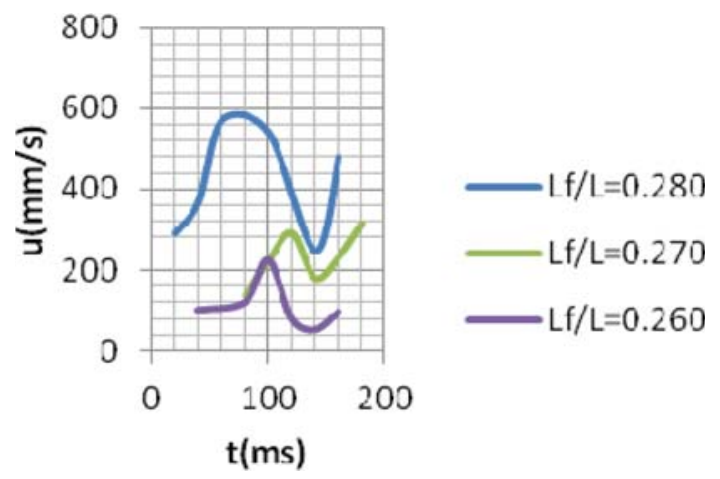

Figure 18: The relationship between velocity and $\mathrm{x}$ axis for trout [21].

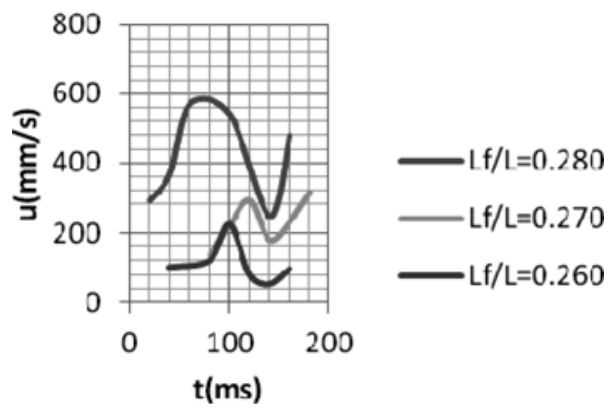

Figure 19: The relationship between velocity and time for different $\mathrm{Lf} / \mathrm{L}$.

Figure 18 shows time-averaged velocity profile of downstream flow component in the wake for a trout by $\mathrm{L}=10 \mathrm{~cm}$ [21]. There is an acceptable consistency between Fig. 16 and Fig. 18 .

Figure 19 reveals the relationship between fish swimming velocity (u) and time of undulatory movement of $P$. sanitwongsei with different $\mathrm{Lf} / \mathrm{L}$. This figure shows that fish swimming velocity increased while $\mathrm{Lf} / \mathrm{L}$ was increasing. Therefore, $\mathrm{u}$ varies undulatory such a sinusoidal form. 
Fish movement was illustrated using a traveling wave [21, 22]:

$$
\mathrm{y}_{\text {body }}(\mathrm{x}, \mathrm{t})=\mathrm{a}(\mathrm{x}) \sin (\mathrm{kx}+\omega \mathrm{t}),
$$

where $\mathrm{y}_{\text {body }}$ is the transverse displacement of body and caudal fin, $\mathrm{k}$ is the number of waves; $\mathrm{k}=2 \pi / \lambda$ and $\lambda$ is the wave length, $\mathrm{x}$ is the displacement along the main axis, $\omega=2 \pi \mathrm{f}, \mathrm{f}$ is the frequency and $t$ is time. Finally $a(x)$ is the second order function which describes wave amplitude where

$$
\mathrm{a}(\mathrm{x})=\mathrm{c}_{2} \mathrm{x}^{2}+\mathrm{c}_{1} \mathrm{x}+\mathrm{c}_{0} .
$$

In this study, $\mathrm{a}(\mathrm{x})$ for $P$. sanitwongsei was analyzed in steady swimming by experimental test. Fish movement was observed and taped. According to the video pictures, pattern of body undulatory movement of fish by $\mathrm{L}=9 \mathrm{~cm}$ were drawn (Fig. 7).

As it was explained earlier, undulatory movement of four $P$. sanitwongse $i$ were studied, where wave length and frequency of body-caudal fin were $0.092 \mathrm{~m}$ and $8.331 / \mathrm{s}$, respectively. Fish movement was considered at four sequences at intervals of $20 \mathrm{~ms}$ and movement trajectories were found. Figure 20 reveals pattern of body-caudal fins undulatory movement of fish by $\mathrm{L}=9 \mathrm{~cm}$ at different times. The velocity of fish swimming was $0.48 \mathrm{~m} / \mathrm{s}$.

To get the time-averaged movement equation, all patterns of body-caudal fins undulatory movement of fish by $\mathrm{L}=9 \mathrm{~cm}$ were put together in Fig. 21. A second-order function was fitted using second-order regression that can explain wave-like motion amplitude. So, the generated equation in this study illustrates the time-averaged movement pattern of caudal fins:

$$
y=-0.1641 x^{2}+0.8774 x-0.8035
$$

\section{CONCLUSIONS AND FUTURE DIRECTIONS}

Natural fish swimming is the best movement in comparison to all under water vehicle. Fish move their body in the way to minimize shear stress and consequently drag forces. The scale of the fish is created to decrease the rough of contact surface and lead to come down shear stress and drag forces. In fish movement, some vortexes are generated behind their bodies, which cause fish propulsion. Therefore, designing a robot fish based on the natural fish swimming would bring brilliant results.

Here, fish movement parameters of four fish were considered and the patterns of body undulatory movement were extracted. The experimental study shows that amplitude of the wave near the head of fish is low and it dramatically increases near its tail. Furthermore, the results reveal that the undulatory movement of caudal fin of fish is almost similar to a sinusoidal form, particularly when $\mathrm{Lf} / \mathrm{L}$ increases. This research resulted in an experimental equation that signifies the relationship between $\mathrm{A} / \mathrm{L}$ and $\mathrm{Lf} / \mathrm{L}$ of fish in steady swimming. It is concluded that fish with high undulatory movement (body and caudal fin), increase in the ratio of the end part to the total length, would lead to an increase in the ratio of amplitude to the total length. In other words, while $\mathrm{Lf} / \mathrm{L}$ goes down, A/L grows up, and hence, both thrust force and displacement increase.

It was revealed at the constant $\mathrm{A} / \mathrm{L}, \mathrm{u} / \mathrm{L}$ increased while $\mathrm{Lf} / \mathrm{L}$ was increasing. Furthermore at the constant amplitude of tail movement with the specified quantity of added mass; as $\mathrm{Lf} / \mathrm{L}$ increases, more velocity needed to generate the same added mass and thrust force.

On the other hand, as Lf/L increases, the velocity of fish swimming and frequency of undulatory movement go up. By increasing the frequency it could be said more vortex would be generated, so the created thrust force would be larger.

It seems that outcomes are also applicable to robotic fish. Therefore, $\mathrm{Lf} / \mathrm{L}$ is an indication for finding the joint point of robotic fish. 
106 A. S. Vaghefi \& M. Abbaspour, Int. J. of Design \& Nature and Ecodynamics. Vol. 9, No. 2 (2014)

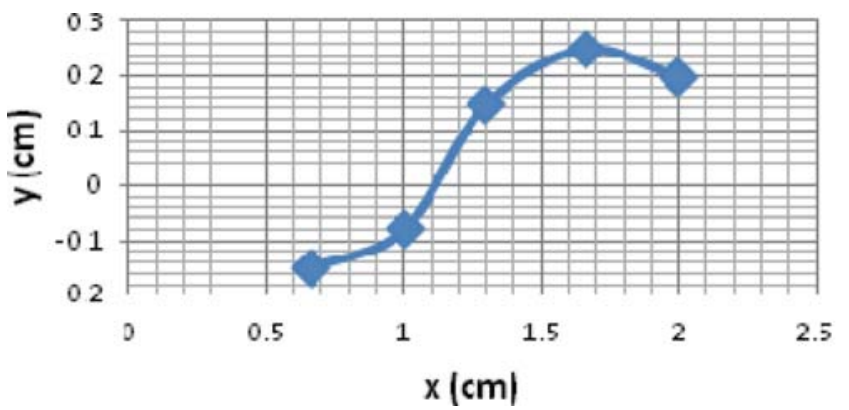

(a)

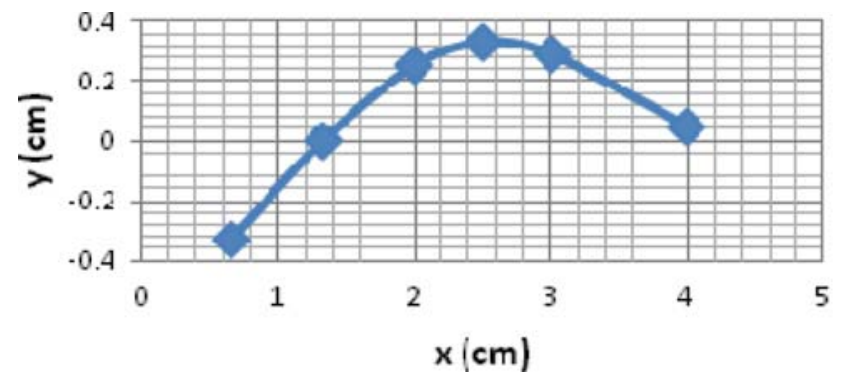

(b)

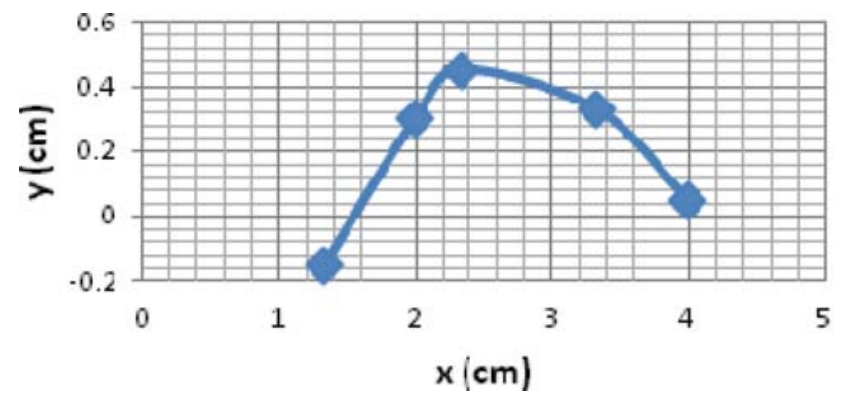

(c)

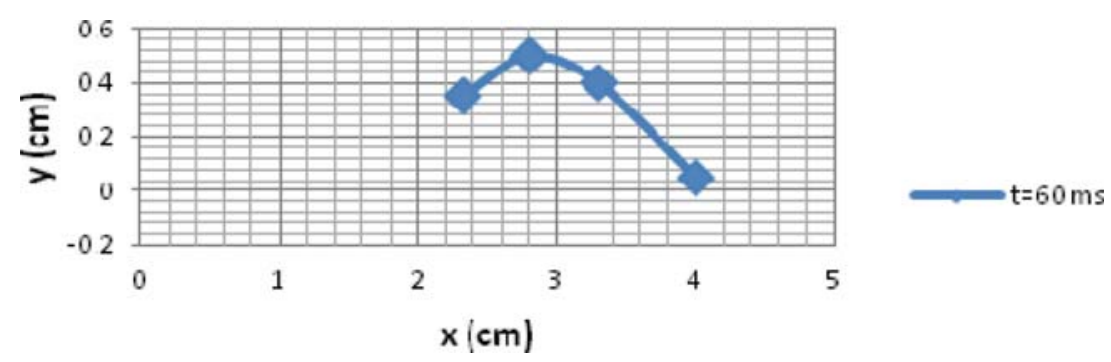

(d)

Figure 20: Pattern of body-caudal fins undulatory movement of Pangasius sanitwongsei by $\mathrm{L}=9 \mathrm{~cm}$ at four sequence $(\mathrm{a}-\mathrm{d})$ at intervals of $20 \mathrm{msec}$. Fish swims at constant velocity, $\mathrm{u}=0.48 \mathrm{~m} / \mathrm{s}$. 


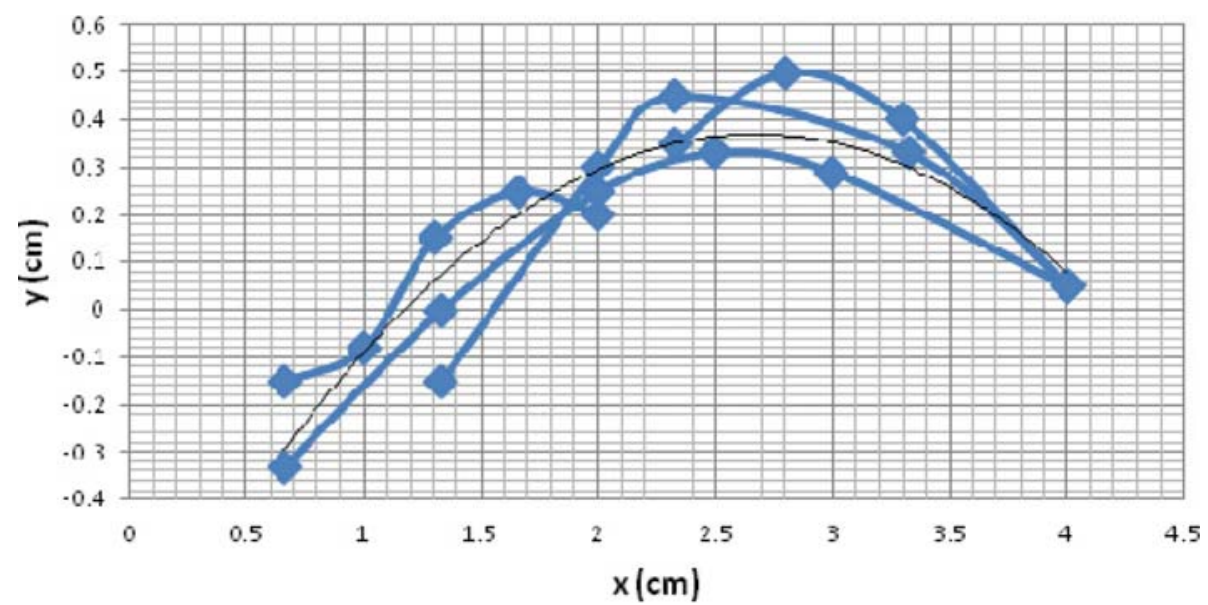

Figure 21: All patterns of body-caudal fins undulatory movement of Pangasius sanitwongsei by $\mathrm{L}=9 \mathrm{~cm}$. Time-averaged movement pattern is presented here.

Also, the movement equation of fish swimming was calculated and a second-order function describing wave amplitude of $P$. sanitwongse $i$ was found. This equation works as a function of a real fish and enables us to design a robotic fish by simulating this equation with servo motors at the joint points.

These equations can be applied for mathematical modeling of robot fishes. Based on these findings, a robotic fish is being made in the laboratory at the IAU (Islamic Azad University). At last, it is recommended for future studies to follow numerical evaluations based on these findings.

\section{REFERENCES}

[1] Arakeri, J.H., Fluid mechanics of fish swimming. Resonance, 14(1), pp. 32-46, 2009. doi: http://dx.doi.org/10.1007/s12045-009-0005-9

[2] Hu, H., Liu, J., Dukes, I. \& Francis, G., Design of 3d swim pattern for autonomous robotic fish. IEEE, International Conference on Intelligent Robots and Systems, pp. 2406-2411, 2006.

[3] Streitlien, G.S. \& Triantafyllou, M.S., Efficient foil propulsion through vortex control. AIAA Journal, 34(11), pp. 2315-2319, 1996. doi: http://dx.doi.org/10.2514/3.13396

[4] Anderson, J.M., Streitlien, K., Barrett, D.S. \& Triantafyllou, M.S., Oscillating foils of high propulsive efficiency. Journal of Fluid Mechanics, 360, pp. 41-72, 1998.doi: http://dx.doi. org/10.1017/S0022112097008392

[5] Lauder, G.V., Nauen, J.C. \& Drucker, E.G., Experimental hydrodynamics and evolution: function of median fins in ray-finned fishes. I. \& C. Biology, 42(5), pp.1009-1017, 2002.

[6] Gillbert, D., Quantitative characterization of three - dimensional pectoral fin kinematics in Bluegill sunfish, Lepomis macrochirus. MURJ, 10, pp. 58-64, 2004.

[7] Lauder, G.V. \& Madden, G.A., Learning from fish: kinematics and experimental hydrodynamics for roboticists. International Journal of Automation and Computing, 4, pp. 325-335, 2006. doi: http://dx.doi.org/10.1007/s11633-006-0325-0

[8] Bejan, A. \& Marden, J.H., Unifying constructal theory for scale effects in running, swimming and flying. Journal of Experimental Biology, 209, pp. 238-248, 2006. doi: http://dx.doi. org/10.1242/jeb.01974 
[9] Bejan, A. \& Marden, J.H., Constructing animal locomotion from new thermodynamics theory. American Scientist, 94, pp. 342-349, 2006. doi: http://dx.doi.org/10.1511/2006.60.1000

[10] Standen, E.M. \& Lauder, G.V., Hydrodynamic function of dorsal and anal fins in brook trout (Salvelinus fontinalis). Journal of Experimental Biology, 210, pp. 325-339, 2007. doi: http:// dx.doi.org/10.1242/jeb.02661

[11] Masalo, I., Reig, L. \& Oca, j., Study of fish swimming activity using acoustical Doppler velocimetry (ADV) techniques. Aquacultural Engineering, 38, pp. 43-51, 2008. doi: http://dx.doi. org/10.1016/j.aquaeng.2007.10.007

[12] Lauder, G.V., Swimming hydrodynamics: ten questions and technical approaches needed to resolve them. Experiments in Fluids, 51, pp. 23-35, 2011. doi: http://dx.doi.org/10.1007/ s00348-009-0765-8

[13] Stafiotakis, M., Lane, D.M. \& Davies, J.B.C., Review of fish swimming modes for aquatic locomotion. IEEE Journal of Oceanic Engineering, 24(2), pp. 237-252, 1999. doi: http://dx.doi. org/10.1109/48.757275

[14] Lauder, G.V. \& Drucker, E.G., Morphology and experimental hydrodynamics of fish fin control surfaces. IEEE Journal of Oceanic Engineering, 29(3), pp. 556-571, 2004. doi: http:// dx.doi.org/10.1109/JOE.2004.833219

[15] Lindsey, C.C., Form function and locomotory habits in fish. Fish Physiology, Vol. 7, Academic Press: New York, pp. 1-100, 1987.

[16] Lauder, G.V., Nauen, J.C. \& Drucker, E.G., Experimental hydrodynamics and evolution: function of median fins in ray-finned fishes. Integrative and Comparative Biology, 42(5), pp.1009-1017, 2002. doi: http://dx.doi.org/10.1093/icb/42.5.1009

[17] Webb, P.W., Simple physical principles and vertebrate aquatic locomotion. American Zoologist, 28, pp. 709-725, 1988.

[18] Takeuchi, S., Kusada, S. \& Kajishima, T., Optimisation of fish shape and swim mode in fully resolved 2-D flow field by genetic algorithm with the least square prediction method. (Chapter13). Bio-mechanisms of Swimming and Flying, eds. N. Kato \& S. Kamimura, Springer-Verlag: Tokyo, pp. 155-166, 2007.

[19] Abbaspour, M. \& Vaghefi, A.S., Experimental hydrodynamics imaging and undulatory movement equation of steady swimming fish (Pangasius sanitwongsei). WIT Transactions on Ecology and the Environment (Design and Nature VI), 160, pp. 171-181, ISSN 1743-3541 (on-line) WIT Press: Southampton, 2012, www.witpress.com, doi:10.2495/DN12016.

[20] Ziegler, M., Iida, F. \& Pfeifer, R., Underwater locomotion: roles of morphological properties and behavioural diversity. Proceedings of the 9th International Conference on Climbing and Walking Robots (CLAWAR), Brussels, Belgium, 2006.

[21] Liao, J.C., Beal, D.N., Lauder, G.L. \& Triantafyllou, M.S., The Kármán gait: novel body kinematics of rainbow trout swimming in a vortex street. Journal of Experimental Biology, 206, pp. 1059-1073, 2003. doi: http://dx.doi.org/10.1242/jeb.00209

[22] Liu, J. \& Hu, H., A 3D simulator for autonomous robotic fish. International Journal of Automation and Computing, 1, pp. 42-50, 2004. doi: http://dx.doi.org/10.1007/s11633-004-0042-5

[23] Vaghefi, A.S. \& Abbaspour, M., Experimental hydrodynamics imaging of Trout in steady swimming. IPCBEE, 32, pp. 135-139, 2012. 